PROGNOSIS

\section{Prediction power}

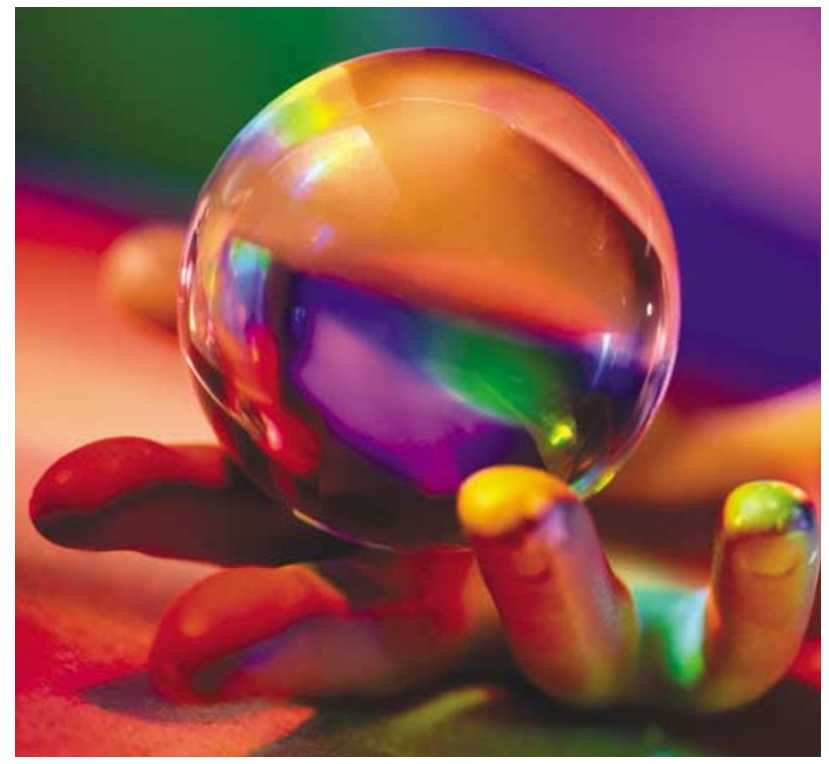

Wouldn't we all like the power to predict the future? For breast cancer patients, the accuracy with which the progress of their disease can be mapped can make the difference between whether they are overtreated or undertreated and can also affect predictions of their survival. Current criteria used for prognosis in breast cancer include age, the size of the tumour, axillary-node status, histological type, pathological grade and hormone-receptor status. van de Vijver and colleagues now add a gene-expression profile to the list of useful prognostic indicators, as they report in the 19 December issue of New England Journal of Medicine.

The authors used a 70-gene prognosis profile that was first described by the same research team earlier in 2002 to classify 295 patients with primary breast cancer - which included 61 of the 78 patients, all lymph-node negative, from the first study — into poor-prognosis and good-prognosis groups. The prognosis signature assigned 115 tumours to the good-prognosis category and 180 to the poor-prognosis category. There was a strong correlation between the probability of remaining free of metastases and of surviving, and the good-prognosis signature.

Interestingly, the prognostic profile was independent of lymph-node status, but was also highly predictive of the risk of distant metastases in the lymph-node-positive subgroup. This could be useful for identifying patients with lymph-node-positive disease who have an unexpectedly good prognosis and indicates that lymph-node metastases develop independently of distant metastases.

The gene-expression profile was also predictive of overall survival $94.5 \%$ of patients in the good-prognosis group survived for 10 years compared with $54.6 \%$ in the poorprognosis group. The hazard ratio for distant metastases in the poor-

\title{
Mass destruction
}

The urokinase pathway is involved in physiological and pathological tissue remodelling processes, including cancer invasion and metastasis. Both urokinase plasminogen activator (uPA) and its receptor ( $\mathrm{UPAR}$ ) are overexpressed in nearly all human cancers, but are only expressed at very low levels in normal tissues, except as a rapid response to tissue injury. Bugge, Leppla and colleagues have exploited this tumour-associated uPA system to activate a targeted immunotoxin, based on anthrax, which causes potent destruction of tumours.

Anthrax toxin is secreted by bacteria as three antigens and is activated when the protective antigen $(\mathrm{PrAg})$ binds to a ubiquitously expressed cell-surface receptor (tumour endothelium marker 8), is cleaved by furin proteases and forms a complex with the two other antigens. Binding of the active part of the complex with fusion protein 59 (FP59) kills the cell by inhibiting translation elongation factor 2 . The authors modified PrAg so that it is cleaved by uPA and not by furin, so that only cancer cells would be targeted.
So, how toxic are these toxins? When just $2 \mu \mathrm{g}$ of the native anthrax toxin, $\operatorname{PrAg}$, was given to mice with FB59, extreme toxicity was observed - widespread organ damage and death occurred within a few hours. By contrast, up to $30 \mu \mathrm{g}$ of the modified toxin, PrAg-U2, resulted in no toxicity. Next, the authors used mice with complete deficiencies in key components of the urokinase pathway - including plasminogen $(\mathrm{Plg})$, which is activated by urokinase - to show that the uPA system is crucial in activation of PrAg$\mathrm{U} 2$. Whereas wild-type mice died when given 40-200 $\mu \mathrm{g}$ PrAg-U2 plus FP59, $u P^{-1-}$, $u P A R^{-1-}$ and $\mathrm{Plg}^{-1-}$ mice remained healthy, and mice null for uPA inhibitor were hypersensitive to the toxin. This shows that the components of the uPA pathway are crucial to the activation of PrAg-U2 in vivo.

The authors now knew that PrAg-U2 was specifically activated by the tumourassociated urokinase system, so they next tested the tumoricidal activity of the toxin in mice bearing established fibrosarcomas, melano-mas and lung carcinomas tumours that have a poor response to conventional treatment. PrAg-U2 reduced the size of the tumours by more than $85 \%$, and completely eradicated all fibrosarcomas in $67 \%$ of mice after only one treatment. No increase in apoptosis was seen, indicating that the toxin triggered necrotic cell death.

The engineered toxin suppressed tumour growth and destroyed established tumours with no toxicity to normal tissues. Because uPA is overexpressed on epithelial, mesenchymal and haematopoietic tumours, this strategy has promise as a broad-acting antitumour therapy. The authors suggest that modifications could be made to increase protease specificity or to use other toxin proteins instead of anthrax. The therapeutic index of immunotoxins already in clinical use might also be improved by adapting this strategy.

Ezzie Hutchinson

(1) References and links

ORIGiNAL ReSEARCH PAPER Liu, S. et al. Potent antitumour activity of a urokinase-activated engineered anthrax toxin. Proc. Natl Acad. Sci. 13 Jan 2003 (doi:10.1073/pnas.0236849100) WEB SITE

WEB SITE

http://wwwdir.nidcr.nih.gov/dirweb/opcb/ptru.asp 